\title{
Phenotypic Characterization of Eosinophilic Esophagitis in a Large Multi-Center Patient Population from the Consortium for Food Allergy Research
}

\author{
Mirna Chehade, MD, MPH ${ }^{1}$, Stacie M. Jones, MD $^{2}$, Robbie D. Pesek, MD², A. Wesley Burks, \\ MD $^{3}$, Brian P. Vickery, MD $^{3}$, Robert A. Wood, MD , Donald Y.M. Leung, MD, PhD ${ }^{5}$, Glenn T. \\ Furuta, $\mathbf{M D}^{6}$, David M. Fleischer, $\mathbf{M D}^{6}$, Alice K. Henning, MS ${ }^{7}$, Peter Dawson, PhD $^{7}$, Robert \\ W. Lindblad, MD , Scott H. Sicherer, $\mathbf{M D}^{1}$, J. Pablo Abonia, $\mathbf{M D}^{8}$, Joseph D. Sherrill, $\mathbf{P h D}^{8}$, \\ Hugh A. Sampson, MD ${ }^{1}$, and Marc E. Rothenberg, MD, PhD $^{8}$
}

\begin{abstract}
Correspondence: Mirna Chehade, MD, MPH, Mount Sinai Center for Eosinophilic Disorders, Jaffe Food Allergy Institute, Icahn School of Medicine of Mount Sinai, One Gustave L. Levy Place, Box 1198, New York, NY 10029, USA, Phone: (212) 241-4880, Fax: (212) 426-1902, mirna.chehade@mssm.edu.

Publisher's Disclaimer: This is a PDF file of an unedited manuscript that has been accepted for publication. As a service to our customers we are providing this early version of the manuscript. The manuscript will undergo copyediting, typesetting, and review of the resulting proof before it is published in its final citable form. Please note that during the production process errors may be discovered which could affect the content, and all legal disclaimers that apply to the journal pertain.

Disclosures:

Mirna Chehade received consulting fees from Shire, Regeneron and Allakos, and research funding from the NIH, PCORI, Nutricia, Regeneron and Shire. Stacie Jones is a member of the Research Advisory Board of the Food Allergy Research and Education (FARE), consultant on the scientific advisory board for Aimmune Therapeutics, received grants for clinical trials from Aimmune Therapeutics, DBV Technologies, Astellas, Inc., FARE, National Peanut Board, and research grant from NIH-NIAID (Immune Tolerance Network), NIH-NIAID (Consortium of Food Allergy Research). She also received payment from EMMES Corporation for CSR Review/Preparation for DBV Technologies. Robbie Pesek reports support through a research training grant as part of the Consortium of Eosinophilic Gastrointestinal Researchers (CEGIR). CEGIR (U54 AI117804) is part of the Rare Disease Clinical Research Network (RDCRN), an initiative of the Office of Rare Diseases Research (ORDR), NCATS, and is funded through collaboration between NIAID, NIDDK, and NCATS. CEGIR is also supported by patient advocacy groups including APFED CURED and EFC. A Wesley Burks received fees as member of the NIH AITC Review Panel, Shareholder in Allertein, from the American Society for Microbiology, as co-editor on food allergy textbook, as Chair and Research Advisory Board for the Food Allergy Research and Education (FARE), and as Board member of the World Allergy Organization. He also received fees as consultant for Adept Field Solutions, consultant and Advisory Board member for Aimmune Therapeutics, and as consultant for Astellas Pharma Global Development, Biomerica, Evelo Biosciences/Epiva Biosciences, First Manhattan Co, Genentech, GLG Research, Insys Therapeutics, Intrommune Therapeutics, PPD Development, Regeneron Pharmaceuticals, Sanofi US Services, SRA International, Stallergenes, UKKO and Valeant Pharmaceuticals North America. He also received research grants from FARE, the NIH, and the Wallace Research Foundation. Brian P Vickery was an employee of Aimmune Therapeutics and had stock options in the company. Robert A Wood has received research grants from the NIH, Astellas, DBV, Aimmune, and HAL-Allergy. Donald YM Leung has no disclosures relevant to this manuscript. Glenn T Furuta is co-Founder for EnteroTrack, consultant for Shire, and received Royalties from UpToDate. David Fleischer is a non-paid board member on the National Peanut Board, FAACT Medical Advisory Board, FARE Clinical Advisory Board. He or his institution has received fees as consultant on the Advisory Board for DBV Technologies, Aimmune Therapeutics, Monsanto, Kaleo Pharma, INSYS Therapeutics, BEFORE Brands, and Intrommune. He received research grants from Nestle Nutrition Institute, DBV Technologies, Aimmune Therapeutics. He received payment for CME lecture and manuscript preparation from Nestle Nutrition Institute, and royalties from UpToDate. Alice K Henning, Peter Dawson and Robert Lindblad are employees of the EMMES Corporation which is a Contract Research Organization (CRO); EMMES was funded by the NIH to be the Statistical and Clinical Coordinating Center for the Consortium for Food Allergy Research (CoFAR). Scott H Sicherer receives grant support from NIAID/NIH and Food Allergy Research and Education. He receives royalties from UpToDate and Johns Hopkins University Press. He receives an honorarium as Associate Editor from the American Academy of Allergy, Asthma and Immunology. J Pablo Abonia has received research grants from the Consortium of Eosinophilic Gastrointestinal Disease Researchers (NIH/NIAID U54AI117804), PCORI SC14-1403- 11593, NIH/NIAID R01 AI124355-01. Joseph D Sherrill has no disclosures relevant to this manuscript. Hugh A Sampson is a part-time employee of DBV Technologies (CSO) and has served as a consultant to Allertein Therapeutics, Hycor and UCB, has received royalties from UpToDate and Elsevier, and funding from NIAID. Marc E Rothenberg is a consultant for Pulm One, Spoon Guru, ClostraBio, Celgene, Shire, Astra Zeneca, GlaxoSmithKline, Allakos, Adare, Regeneron and Novartis and has an equity interest in the first four listed and Immune Pharmaceuticals, and royalties from reslizumab (Teva Pharmaceuticals) and UpToDate. He is also an inventor of patents, owned by Cincinnati Children's.
\end{abstract}


${ }^{1}$ Icahn School of Medicine at Mount Sinai, New York, NY

2University of Arkansas for Medical Sciences and Arkansas Children's Hospital, Little Rock, AR

${ }^{3}$ University of North Carolina at Chapel Hill, Chapel Hill, NC

${ }^{4}$ Johns Hopkins University Medical Center, Baltimore, MD

${ }^{5}$ National Jewish Health, Denver, CO

${ }^{6}$ Children's Hospital Colorado, University of Colorado School of Medicine, Aurora CO

${ }^{7}$ The EMMES Corporation

${ }^{8}$ Division of Allergy and Immunology, Cincinnati Children's Hospital Medical Center, Cincinnati, $\mathrm{OH}$

\section{Abstract}

Background-Eosinophilic esophagitis (EoE) is increasingly common, but data on phenotypic aspects are still incomplete.

Objectives-To describe the clinical, endoscopic and histopathological features of a large number of children and adults with EoE across the US.

Methods-This was a multi-site single visit registry enrolling subjects aged 6 months-65 years with EoE. Participants provided responses regarding their medical history, with verification of the diagnosis and history by the study teams.

Results-705 subjects were analyzed (median [IQR] age at enrollment 11.2 [6.7-17.7] years, $68.2 \%$ male, $87.9 \%$ whites). 67 subjects had concurrent gastrointestinal eosinophilia, with gastric mucosa most common. An age and race-dependent time gap was present between symptom onset and time of diagnosis (adults and whites with longer gap). Food allergy and atopic dermatitis were associated with a decrease in this gap. Symptoms varied with age (more dysphagia and food impaction in adults) and with race (more vomiting in non-whites). Esophageal rings and strictures at diagnosis were more common in adults, although esophageal eosinophilia was comparable among age groups. Concomitant allergic disease (91\%), infectious/immunological disorders (44\%), neurodevelopmental disorders (30\%) and failure to thrive (21\%) were common. Depression/anxiety increased with age. EoE was reported in $3 \%$ of parents and $4.5 \%$ of siblings.

Conclusions-Gastrointestinal eosinophilia is present in $~ 10 \%$ of EoE patients; the symptomdiagnosis time gap is influenced by age, race, food allergy and atopic dermatitis; symptoms vary with race; concurrent infectious/immunological disorders and mental health disorders are common; and the level of esophageal eosinophils is comparable in patients with and without fibrostenotic features.

\section{Keywords}

Eosinophilic esophagitis; atopy; autoimmune disease; registry; multi-site; eosinophilic gastritis; eosinophilic gastroenteritis; eosinophilic colitis; food allergy; proton pump inhibitor; race 


\section{Introduction}

Eosinophilic esophagitis (EoE) is a chronic immune-mediated disease of the esophagus characterized by symptoms related to esophageal dysfunction, and by an eosinophilpredominant esophageal inflammation. ${ }^{1}$ Symptoms vary by patient age, and include abdominal pain, emesis, gastroesophageal reflux (GER) symptoms, dysphagia, esophageal food impactions, and failure to thrive. ${ }^{1}$ EoE is triggered by foods and possibly environmental allergens. ${ }^{2,3}$ Prevalence of EoE in the United States (US) has been steadily increasing, with estimates currently at $0.5-1$ case/1000 persons. ${ }^{4}$ Data on phenotypic aspects of this disease are lacking or incomplete because most reports are derived from single centers 5,6 where data are limited by the small size of the population studied, biases in which the data is recorded and/or interpreted, and local referral biases at specific institutions or clinical sites, which collectively lead to uncertainty about generalizability.

In this study, we describe the clinical, endoscopic and histopathological features of a large number of children and adults with EoE across the US using a multi-site registry established by the Consortium for Food Allergy Research (CoFAR). The advantages of this cohort compared with prior studies on EoE include the following: (1) large cohort evaluated at 5 US sites, (2) regulatory supervision with independent site monitoring to ensure accuracy of data; (3) inclusion of children and adults in the same study population; and (4) a detailed medical and family history. These unique features allowed a more accurate definition of EoE phenotypes across age groups, as well as discovery of potential disease associations that would not be possible otherwise. In addition, the multi-site study allowed us to capture subjects with a wide range of ages, racial backgrounds, geographical locations and socioeconomic conditions. This heterogeneity allowed us to make several important observations.

\section{Methods}

This was a multi-site, single visit registry of subjects 6 months to 65 years of age of any race, gender or ethnicity, with documented EoE, diagnosis based on histology demonstrating peak intraepithelial esophageal eosinophils $\geq 15$ / high power field (HPF) and symptoms of esophageal dysfunction. Subjects were recruited from the 5 US sites forming CoFAR: Icahn School of Medicine at Mount Sinai, New York, New York; Duke University Medical Center, Durham, North Carolina (with the investigative team having relocated as of March 2012 to the University of North Carolina, Chapel Hill, North Carolina); National Jewish Health, Denver, Colorado; University of Arkansas for Medical Sciences and Arkansas Children's Hospital, Little Rock, Arkansas; and Johns Hopkins University School of Medicine, Baltimore, Maryland.

This registry was part of a study conducted by CoFAR, the primary aim of which was to establish the genetic basis of EoE, and was conducted in conjunction with the Cincinnati Children's Hospital Medical Center, which performed the genetic analysis on blood or saliva samples collected from the subjects at the time of enrollment. ${ }^{7}$ 
Participants provided responses regarding their medical history, with verification of the EoE diagnosis and history by the study teams. Participants with concurrent eosinophilia at gastrointestinal sites in addition to the esophagus were permitted entry into the study, since these patients were thought to have EoE as their primary gastrointestinal disease. This was recorded based on the investigator's judgment at each participating site based on history and local review of the pathology reports. It could be argued that these patients have eosinophilic gastroenteritis or colitis with esophageal involvement rather than EoE, but the patients presented with esophageal symptoms and were clinically diagnosed with EoE. Furthermore, we decided that new and potentially interesting findings would be discovered by allowing more broad inclusion criteria into the study. In addition, resistance to proton pump inhibitor (PPI) therapy was recorded but not required for enrollment. Patients with possible PPIresponsive esophageal eosinophilia (PPI-REE) were not excluded for the following reasons: 1) Since our study allowed recruiting patients at any time after their EoE diagnosis was made, we did not want to exclude subjects diagnosed before 2007 (when the consensus recommendations specified requiring PPI-responsiveness testing or $\mathrm{pH}$ probe testing for the diagnosis of EoE) ${ }^{8}$ who were historically proven to have EoE by demonstrating histological response to dietary interventions alone; 2) Since this analysis was part of a larger genetic study, we did not want to exclude these patients because we had interest in determining whether these patients had any genotypic similarities/differences from the remainder of the population. More recently, preliminary data demonstrated that patients with PPIREE are similar to those with classic EoE (resistant to PPI therapy) given their favorable response to EoE-specific therapies, as well as evidence of a similar esophageal gene expression profile. 9-11

Exclusion criteria included known causes of esophageal eosinophilia other than EoE, and hypereosinophilic syndrome. Data were obtained using questionnaires with predominantly closed-ended questions that were uniformly used at all sites. De-identified data were then transferred by study staff to a password-protected web-based data entry system managed by the Statistical and Clinical Coordinating Center (The Emmes Corporation, Rockville, MD), which allowed enrollment at all participating sites in a uniform manner. As data were entered, they were validated through range and within-form consistency checks, and duplicate enrollment was prevented in the event a subject had travelled among sites for medical care.

The following information was collected: demographics, medical and surgical history (including review of systems, history of extra-esophageal gastrointestinal eosinophilia, symptoms, self-reported food allergies), and family history. Information was provided by the patients. In addition, histopathological data were collected at each site, as well as endoscopic findings on the corresponding procedure when available.

The study was approved by the institutional review board of each participating center. Written consent and, when applicable assent, were obtained from all participants. The questionnaires employed can be found in this article's Online Repository at www.jaciinpractice.org. 


\section{Statistical analysis}

The primary purpose of this study was to establish a genetic basis for EoE and clinical/ demographic information were collected as part of that study. As such, there were no prespecified analyses for phenotypic data. Rather, analysis was primarily descriptive, with continuous variables assessed as medians and interquartile ranges [IQR] and categorical variables described as proportions. Factors were described by age categories at enrollment, and formal hypothesis testing evaluated differences between those age groups using Kruskal-Wallis tests (Wilcoxon Rank sum for pairwise group comparisons) for continuous variables or Fisher's Exact test for categorical variables. Similar analyses were performed comparing whites to non-whites, study sites, and those with and without a greater than 6week course of high dose PPI. Age groups were defined prior to any analysis as $<11$ years old, 11 to 17 years old, and adults $\geq 18$ years old. Esophageal eosinophil counts per HPF were truncated at 100 per data collection procedures. Participants reporting counts that were not a specific number but were indicated only as being greater than a number less than 100 were excluded from the eosinophil count analysis. This was done to allow comparability of values through 100. Comparison of eosinophil counts between endoscopic findings was performed via Wilcoxon Rank-Sum test. As the analysis was primarily descriptive, significance was assessed at the 0.05 level. Analysis was performed in SAS v9.3.

\section{Results \\ Demographics}

708 subjects were enrolled between May 2011 and March 2016, and 705 subjects were analyzed, as 3 were determined to be ineligible. The highest number of participants was enrolled from New York (226 subjects, 32.1\%), followed by 142 from Arkansas (20.1\%), 127 from North Carolina (18.0\%), 110 from Maryland (15.6\%) and 100 from Colorado $(14.2 \%)$. Age at enrollment ranged from 0.9 to 56.2 years, with a median [IQR] of 11.2 [6.7-17.7] years. The majority of the subjects were male (68.2\%) and predominantly Whites (87.9\%), with 7.1\% Black and 2.8\% Asian. Household income covered the entire spectrum but the largest group was $\geq \$ 100,000$ (276 subjects), though 235 subjects declined to give a response. A large number reported having private medical insurance $(601 / 703=85.5 \%)$. More detailed site-specific demographic characteristics are listed in table E1 available in this article's Online Repository at www.jaci-inpractice.org.

\section{Diagnosis}

$54.8 \%$ had confirmed receiving PPI therapy for more than 6 weeks or a normal $\mathrm{pH}$ probe as part of their diagnostic work-up for EoE. No significant differences in age at enrollment and age at diagnosis were found between patients who were treated with PPI and those who did not receive PPI as a diagnostic confirmation therapy or were unsure (median [IQR] age at enrollment 10.7 [6.9-16.9] versus 11.9 [6.5-18.8] years, $\mathrm{p}=0.26$ respectively; median [IQR] age at diagnosis $8.0[3.0-15.0]$ versus 8.0 [3.0-16.0] years, $\mathrm{p}=0.69$ respectively). Furthermore, no significant difference was found between the two groups with respect to gender, symptoms and co-morbidities (table E2, available in this article's Online Repository at www.jaci-inpractice.org). A notable exception was concurrent gastroesophageal reflux disease (GERD). The group that did not have PPI confirmation had significantly fewer 
reported diagnoses of GERD than the PPI-confirmed EoE group (47.6\% no PPI versus $59.6 \%$ PPI, $\mathrm{p}=0.002$ ), suggesting that exclusively GERD patients with esophageal eosinophilia are not overly influencing our non-confirmed group.

Extra-esophageal eosinophilia was most commonly reported in the gastric mucosa; 37/705 (5.2\%) had concurrent eosinophilic gastritis versus 4/705 (0.6\%) with eosinophilic enteritis and 12/705 (1.7\%) with eosinophilic colitis. Eosinophilic infiltration of more than one gastrointestinal site besides the esophagus was rare, with 14/705 (2.0\%) of subjects having this diagnosis (table 1). In total, 67 patients (9.5\%) had eosinophilia at other sites, such as the gastric, duodenal, or colonic mucosae, including 14 with eosinophilia at more than one gastrointestinal site (table 1). This percentage was comparable when subjects were compared with respect to race (table 1).

Median [IQR] age at diagnosis for the entire cohort was 8.0 [3.0-15.0] years and 5.0 [1.0$12.0]$ at symptom onset. Among children $<11$ years of age, median [IQR] age at diagnosis was 3.0 [1.9-6.0] years and 1.1 [0.4-3.0] years at symptom onset. Patients 11-17 years of age had a median [IQR] age at diagnosis of 12.0 [9.0-13.0] years and 10.0 [4.0-12.0] years at symptom onset. Adults ( $\geq 18$ years) had a median [IQR] age at diagnosis of 29.0 [18.039.0] years and 19.0 [12.0-30.0] years at symptom onset. These large time gaps between symptoms and diagnosis were more pronounced with increasing age at enrollment; median [IQR] time from symptom onset to diagnosis was 4.0 [1.0-12.0] years in adults, compared to 2.0 [1.0-4.0] years in patients 11-17 years of age and $1.0[0.5-2.3]$ in children $<11$ years of age $(\mathrm{p}<0.0001)$. This delay was also racedependent, as whites had longer time from symptom onset to diagnosis; median [IQR] time gap 1.8 [0.7-4.0] years compared to 1.0 $[0.5-2.0]$ years in non-whites, $\mathrm{p}=0.005$.

The time gap between onset of symptoms and diagnosis was independent of concurrent eosinophilia at other gastrointestinal sites, as we found no difference between those with EoE alone (90.5\% of subjects) and those with EoE and concomitant eosinophilia at other sites of the gastrointestinal tract ( $9.5 \%$ of subjects) $(\mathrm{p}=0.26$ for age at diagnosis, and $\mathrm{p}=0.54$ for age at $\mathrm{EoE}$ symptom onset, and $\mathrm{p}=0.48$ for time from symptom onset to diagnosis; table 1). We further evaluated whether various patient characteristics such as gender, atopic history, or family history of EoE can influence this significant diagnostic delay (table 2). We found that history of food allergy or atopic dermatitis was associated with a significantly shorter time gap between symptom onset and diagnosis. However, no differences were observed with respect to gender (median time gap 1.4 [IQR 0.6-4.0] years in males versus 1.9 [IQR 1.0-5.0] years in females), personal history of other atopic diseases, or even family history of EoE (table 2). History of suspected drug-induced anaphylaxis was associated with a significantly longer diagnostic delay of EoE (table 2).

\section{Symptoms}

Subjects reported a variety of symptoms experienced within the previous 12 months prior to enrollment (table 3). These included abdominal pain (53.6\%), dysphagia (50.5\%), nausea (40\%), emesis (40\%), esophageal food impaction (38.1\%), heartburn (37.2\%), diarrhea (32.1\%), chest pain (21.1\%), and bloody stools (7.5\%), with most subjects reporting multiple symptoms. Abdominal pain, emesis, heartburn and chest pain, as well as dysphagia 
and food impaction differed significantly among age groups (table 3). Abdominal pain and emesis were observed to be higher in children <11 years of age and 11-17 years of age than adults; whereas heartburn, chest pain, dysphagia and food impactions were observed to be lowest in children < 11 years of age and appeared to increase with age. Nausea, diarrhea and bloody stools were comparable among the 3 age groups. Bloody stools were not limited to subjects with EoE with concurrent gastrointestinal eosinophilia, but were also reported in subjects with EoE alone (6.6\% of subjects with EoE alone versus $16.4 \%$ of subjects with $\mathrm{EoE}$ and eosinophilia at other gastrointestinal sites, $\mathrm{p}=0.01$ ). Of the 42 subjects that had $\mathrm{EoE}$ alone without concurrent gastrointestinal eosinophilia and had bloody stools, only one patient had Crohn's disease. We further compared symptoms between subjects with EoE alone to those with EoE and eosinophilia at other gastrointestinal sites and found those with EoE alone had significantly less abdominal pain $(51.3 \%$ EoE alone versus $74.6 \%$ EoE with GI eosinophilia, $\mathrm{p}=0.0003)$ and significantly more esophageal food impactions (39.6\% EoE alone versus $23.9 \%$ EoE with GI eosinophilia, $\mathrm{p}=0.01$ ); no differences were found in nausea, emesis, heartburn, chest pain, gastroesophageal reflux, dysphagia, or diarrhea. When symptoms were compared with respect to race, dysphagia, esophageal food impactions and heartburn were significantly more common in whites, while emesis was significantly more common in non-whites (table 3 ).

Food impactions requiring urgent endoscopic removal were reported in $6.1 \%$ of subjects; median [IQR] age of these patients was 26.0 [15.2-41.1] years. Esophageal stricture was seen on endoscopy in $10.1 \%$ of subjects; median [IQR] age was 33.2 [19.9-42.4] years. Esophageal dilation was reported in $9.9 \%$ of subjects; median [IQR] age was 35.0 [22.144.3] years.

When the diagnostic endoscopy was analyzed, esophageal white plaques and furrows were the most common features reported (table 3). Esophageal rings and strictures at diagnosis were significantly different between age groups and were observed to be more common in adults than in patients $<11$ years of age and 11-17 years of age, while all other features, including inflammatory features (furrows, white plaques and loss of vascular pattern) were comparable (table 3). Consistent with the latter endoscopic finding, esophageal eosinophilia was comparable among the age groups; median peak esophageal eosinophil counts (from the distal, mid, and proximal esophagus) were 50/HPF [IQR 33-84] in children and 55/HPF [IQR 40-90] in adults, $\mathrm{p}=0.30$. Peak eosinophil counts in those with endoscopic inflammatory features (furrows, white plaques, or loss of vascular pattern) were higher than those without these features (median 60/HPF [IQR 40-100] versus 40/HPF [IQR 29-70], $\mathrm{p}<0.0001)$. However, peak eosinophil counts in patients with and without fibrostenotic features (rings, stricture or shearing) were comparable (median 60/HPF [IQR 40-93] versus 55/HPF [IQR 34-100], p=0.25).

Endoscopic features were evaluated in relation to the subjects' race. Esophageal rings, but not strictures, were significantly more often reported in whites. However, endoscopic inflammatory features were comparable among race (table 3 ) as were peak esophageal eosinophils/HPF (median 50/HPF [IQR 35-85] in whites versus 50/HPF [IQR 35-75] in non-whites, $\mathrm{p}=0.50$ ). 


\section{Medical history}

Concomitant medical and surgical histories were obtained and are summarized in table 4. As expected, ${ }^{8}$ concurrent GERD was very commonly reported (54.2\%). While other gastrointestinal diseases were present including lower gastrointestinal disorders, they were similar in prevalence to that of the general population except for celiac disease reported in $2.0 \%$ of subjects, which appeared slightly higher than expected for the general population $(0.3-1 \%) .{ }^{12}$ Notably, concurrent inflammatory bowel disease (Crohn's and ulcerative colitis) was found in a total of 11 subjects with median [IQR] age of 19.8 [14.5-26.0] years; these subjects did not have eosinophilia in any other GI site besides the esophagus. Irritable bowel syndrome was significantly more common in adults.

Allergic disorders were reported in the majority of subjects (90.6\%), both in adults and children (table 4). Food allergy was most commonly reported $(67.0 \%)$ followed by allergic rhinitis $(60.3 \%)$, atopic dermatitis $(46.4 \%)$, asthma (45.4\%), allergic conjunctivitis (31.2\%), urticaria (26.2\%), food anaphylaxis (27.1\%) and angioedema (13.2\%). Food allergies, food anaphylaxis, asthma and allergic skin disorders (atopic dermatitis and urticaria) had significant differences among age groups and were observed to be higher in children $<11$ years of age and 11-17 years of age compared to adults, while allergic rhinitis and conjunctivitis did not differ between age groups. Nonwhites had significantly higher rates of food allergy and atopic dermatitis (table 4), both of which were associated with a shorter gap between symptom onset and diagnosis in the overall population (table 2).

Subjects in this study also reported a high prevalence of infections (44.4\%), such as sinusitis (28.5\%) and pneumonia (24.5\%). Surgeries related to these disorders were also common, including tympanostomy tube placement (19.6\%). Autoimmune disease was also reported in $4.7 \%$ of subjects, which appeared comparable to what is reported in the general population (3.2\%). ${ }^{13}$ Interestingly, autoimmune disease was only reported in white subjects (table 4). Depression/anxiety was more prevalent than what is known for the general population, ${ }^{14}$ and was significantly more often reported with increasing age (24.0\% in adults compared to $9.3 \%$ in children < 11 years of age, and $19.0 \%$ in those $11-17$ years of age, $\mathrm{p}<0.0001$ ). Failure to thrive was also common ( $21.3 \%$ of subjects), and was significantly different among age groups with it being more frequently reported in the younger population $(\mathrm{p}<0.0001) .5 .8 \%$ of subjects underwent gastrostomy tube placement, mostly children (table 4). $29.6 \%$ had history of neurological/developmental disorders. Specifically, $6.8 \%$ of the EoE patients had a history of prematurity, significantly higher in non-white individuals (12.9\% non-white vs. $6.0 \%$ white, $\mathrm{p}=0.03$ ) (table 4$)$; there was also a high rate of other comorbidities such as developmental delay (11.2\%).

While subjects reported IgE-mediated allergy to multiple foods known as common allergens such as milk, egg, wheat, soy, peanut, tree nuts, fish, and shellfish (table E3, available in this article's Online Repository at www.jaci-inpractice.org), high rates of allergy were also reported to less common allergens such as beef, poultry and corn. Interestingly, these allergies were not limited to young children as would be expected for less common allergies,

15 but were also present in older children and adults. 


\section{Family History}

Parents and siblings of subjects with EoE had a high prevalence of atopic diseases including asthma, allergic rhinitis, atopic dermatitis and urticaria, as well as food allergy (table E4, available in this article's Online Repository at www.jaci-inpractice.org). Family history of EoE was also more common than that expected for the general population $(0.5-1 / 1000):^{4}$ $3.0 \%$ of subjects had $\geq 1$ parent with a history of EoE, $4.5 \%$ had $\geq 1$ sibling with a history of EoE, and $6.2 \%$ had $\geq 1$ parent and/or sibling with a history of EoE. In addition, 8.1\% reported having $\geq 1$ parent with food impaction and 5.2\% having $\geq 1$ parent who had esophageal dilation. This difference in the prevalence of EoE versus the symptoms of food impaction and esophageal dilations was not found in siblings. GERD was also more prevalent in parents than in siblings of subjects with EoE. In addition to EoE, eosinophilic gastrointestinal disorders (EGID) besides EoE were surprisingly prevalent in family members, given that EGIDs are even less prevalent than EoE in the general population, with $2.9 \%$ having $\geq 1$ parent with EGID, 2.9\% having $\geq 1$ sibling with EGID, and 4.8\% having $\geq 1$ parent and/or sibling with EGID. An increased rate of other immune diseases was noted in parents of subjects with EoE ( $7.9 \%$ had $\geq 1$ parent with inflammatory bowel disease, $6.9 \%$ had $\geq 1$ parent with autoimmune disease, and $1.4 \%$ had $\geq 1$ parent with celiac disease), all these being higher than expected for the general population. ${ }^{12,13,16}$ (table E4, available in this article's Online Repository at www.jaci-inpractice.org)

\section{Discussion}

In this multi-center registry of subjects with EoE that included an extensively detailed medical history, we were able to capture subjects with a wide range of ages, racial backgrounds, geographical locations and socioeconomic conditions. This heterogeneity allowed us to make several important observations. Amongst these, the following findings have been described for the first time or have been solidified in this study: (1) gastrointestinal eosinophilia at the site contiguous to the esophagus occurs in $\sim 5 \%$ of patients; (2) a large time gap (1.5 years) between symptom onset and disease diagnosis exists, and this gap is influenced by age (adults had a longer time from symptom onset to diagnosis than children), race (whites had a longer time from symptom onset to diagnosis) and history of food allergy or atopic dermatitis (both associated with a decrease in the time gap); (3) symptoms vary with age (non-specific gastrointestinal symptoms were more common in children, and esophageal symptoms such as heartburn, chest pain and dysphagia were common in adults); (4) symptoms vary with race (dysphagia, esophageal food impactions and heartburn were significantly more common in whites, while emesis was significantly more common in non-whites); (5) failure to thrive was common (21.3\% of subjects) especially in the younger population; (6) infectious/immunological disorders including celiac disease were prevalent in EoE subjects and their family members; (7) depression and anxiety had significant differences between age groups of EoE patients and were observed to increase with age (for example, $24.0 \%$ in subjects $\geq 18$ years of age compared to $9.3 \%$ in children $<11$ years of age); (8) there was a high prevalence of neurodevelopmental disorders including prematurity in the EoE population; (9) peak esophageal eosinophil counts in patients with and without fibrostenotic features (rings, stricture or shearing) were comparable, suggesting that fibrostenotic complications are not 
necessarily an end-stage component of the disease, and pointing towards the importance of concurrently treating the underlying esophageal inflammation when performing esophageal dilation in patients with fibrostenosis; and (10) family history of EoE and EGID is present in a relatively large fraction of EoE patients $(6.2 \%$ and $4.8 \%$ with $\geq 1$ parent and/or sibling respectively), consistent with a strong hereditary component as now observed in this multisite large cohort.

We were able to confirm the predominance of males among those with EoE, and the fact that EoE affects patients of all races. ${ }^{1,17}$ We observed for the first time that concurrent eosinophilic gastritis is not uncommon in patients with esophageal eosinophilia and its related symptoms, with $5.2 \%$ of our subjects diagnosed with eosinophilic gastritis. Previous studies have examined the reverse association, i.e. the prevalence of esophageal eosinophilia in patients with eosinophilic gastritis. In a study by Jensen et al examining children and adults, ${ }^{18} 10.6 \%$ of the EG patients had concurrent EoE, although these data were derived from a large insurance database and therefore could not be verified. In a single-center pediatric study by Ko et al, ${ }^{19}$ where all data were verified and biopsies were re-evaluated by the investigators, $43 \%$ of the patients with EG had concurrent EoE. In addition, Caldwell et al found that $~ 90 \%$ of EG patients had extra-gastric involvement with the esophagus being the most commonly involved secondary organ. ${ }^{20}$ This association of esophageal and gastric eosinophilia is important to emphasize, since it highlights that the vast majority of EoE patients with extra-esophageal eosinophilia have only gastric involvement and rarely hypereosinophilic syndrome and/or other extra-esophageal disease. Nevertheless, it is important for clinicians to be aware of the association, necessitating the consideration of endoscopic biopsies of the stomach and duodenum in EoE patients at the time of diagnosis, and possibly at follow-up, ${ }^{21}$ as finding gastrointestinal eosinophilia beyond the esophagus may influence management decisions.

We found a large time gap between symptom onset and the diagnosis of EoE. This gap was independent of concurrent gastrointestinal eosinophilia at other sites. The time gap was agedependent, however, as it increased with increasing patient age at diagnosis. We also found that food allergy and atopic dermatitis were associated with a more prompt diagnosis. Food allergy and atopic dermatitis were more common in the younger population, therefore potentially influencing the variability of the diagnostic time gap with age. We also found that the time gap was race-dependent, with whites having a longer gap between symptom onset and diagnosis. But again here, non-white subjects had significantly more food allergy and atopic dermatitis, which could have also influenced this finding. One potential explanation for food allergy and atopic dermatitis influencing the time gap from symptoms to diagnosis may be that clinicians are aware of EoE being associated with allergic disease, ${ }^{1}$ and are including it in their differential diagnosis when gastrointestinal complaints are noted. Interestingly, family history of EoE was not associated with a shorter time gap to diagnosis, likely because parents of EoE subjects often have their EoE diagnosis made after, not before, their children get diagnosed; as they discover through their children's medical visits that their chronic esophageal symptoms are also suggestive of EoE. This observation stresses the importance of increasing knowledge about the disease, particularly focusing on the strong hereditability of EoE. Furthermore, a large percentage of our subjects had private health insurance, which tends to be linked to easier access to care. Therefore, it is possible that the 
time gap between symptom onset and diagnosis of EoE is even longer in the general population.

Adult subjects predominantly presented with dysphagia and esophageal food impactions rather than abdominal pain, nausea and emesis. Dysphagia in EoE is known to be a slowly progressive symptom, often with behavioral feeding modifications made by patients to prevent worsening of dysphagia and esophageal food impactions. ${ }^{22}$ Due to the progressive nature of the disease, patients may develop compensatory eating behaviors, potentially leading to a delay in seeking medical attention and proper diagnosis. Similar findings were obtained in a study of Swiss EoE adult patients, where the median diagnostic delay in adults was twice that in children, with characteristics such as EoE family history and atopic diseases/allergies not found to be risk factors for the increased length of diagnostic delay. ${ }^{23}$

Abdominal pain, emesis and dysphagia were some of the most common symptoms reported in our study. Dysphagia and esophageal food impactions, in addition to gastroesophageal symptoms including heartburn, were more common in whites, and non-whites reported more emesis. Our findings validate a large retrospective multicenter study ${ }^{17}$ in which whites had more dysphagia, food impactions and classic features such as esophageal rings than nonwhites, although heartburn was less common in whites in that study. It is notable that genetic analysis of EoE has been limited to whites, ${ }^{7}$ calling attention to the importance of examining the genetic basis of non-whites as sample sizes grow. In addition, we confirmed previous reports ${ }^{5,24}$ of a high prevalence of failure to thrive in EoE, especially in the younger population, which necessitates future in-depth evaluation.

Interestingly, lower gastrointestinal symptoms including diarrhea and bloody stools were commonly reported by our subjects. While the accuracy of these reports was limited by the absence of strict definitions for each symptom in the questionnaire, these observations warrant further investigation. Diarrhea has been reported previously in children with EoE, ${ }^{24}$ and intestinal permeability has been reported to be increased in patients with EoE. ${ }^{25}$

Around $15 \%$ of subjects in this study reported depression/anxiety, which increased in prevalence with age. Depression/anxiety and decreased quality of life have been reported previously in EoE. ${ }^{26,27,28}$ Our data suggest that a more comprehensive approach that includes mental health support should be considered in the management of patients with EoE. Furthermore, neurodevelopmental disorders were reported in our subjects and deserve particular attention. EoE and developmental disorders have been described previously, ${ }^{29}$ and pediatric phenome-wide associated studies point to a potential link between EoE and these disorders. ${ }^{30}$

Celiac disease was slightly more prevalent in EoE subjects than expected for the general population. Although the association between EoE and celiac disease remains unclear as the two diseases are currently thought to be likely independent, ${ }^{31}$ our finding warrants further investigation. Autoimmune diseases were reported in our subjects (4.7\%) and their family members. This finding was limited to white subjects, raising the suspicion for a different disease phenotype in this patient subset. Further studies are warranted for this subgroup, since findings from these results may influence future medical therapies for these patients, as 
shared immunopathology may be present. A very similar result was found in a single population-based cohort study in Utah, a predominantly white cohort of northern European descent, where a high rate of both personal and family histories of autoimmune disorders was found. ${ }^{32}$ In addition to atopic diseases in both subjects and their families, subjects with EoE also reported a high rate of pneumonia and sinusitis, which can possibly be secondary to poorly controlled allergic rhinitis and asthma, and immunodeficiency. These concurrent immune-related/infectious diseases suggest the possibility of a systemic immune dysregulation in EoE beyond its localization to the esophagus, and is worth further investigation since immunodeficiency has been reported previously in patients with significant esophageal eosinophilia. ${ }^{33,34}$

Lastly, EoE and EGID were common in family members of patients with EoE. Interestingly, the prevalence of food impactions and esophageal dilatations was higher than the reported prevalence of EoE in parents, while this difference was not seen in siblings. This observation in parents is possibly secondary to an increase in EoE awareness over time, leading to a diagnosis of EoE in children more readily than in adults, while siblings, who are younger, may not yet have developed these esophageal complications, or they were adequately treated as therapy options increased with the next generation. In addition, GERD was more prevalent in parents than in siblings of our subjects with EoE, with esophageal food impactions and dilations in that case being potential complications of GERD rather than EoE in some of the parents.

Our study has several limitations. We decided to include patients who did not have PPI therapy as a requirement for EoE diagnosis. Therefore, patients with PPI-REE were not excluded in this study. Recent research, however, is identifying patients with PPI-REE as having EoE. In fact, the European Society of Eosinophilic Oesophagitis established PPIREE as EoE in their new guidelines, therefore eliminating the concept of PPI-REE as a separate entity, ${ }^{35,36}$ based on evidence of similar gene expression profiles in EoE and PPIREE, ${ }^{10,11}$ demonstration of an anti-inflammatory effect of PPI,${ }^{37}$ and reports of patients that interchangeably respond to PPI and dietary eliminations targeted for EoE patients. ${ }^{9}, 38$ Based on the above, we do not believe that GERD is the cause of PPIREE. We believe that patients with GERD accounting for esophageal eosinophilia were not included in this study, since all patients included had their diagnosis confirmed by the investigators, i.e. other causes of esophageal eosinophilia including GERD were ruled-out. In addition, our study is limited by self-report of co-morbidities, although research coordinators verified the information provided by medical record review as available. Lastly, assessment of gastrointestinal eosinophilic disease beyond the esophagus was limited by the fact that no definitions yet exist for eosinophilic gastritis, enteritis and colitis, therefore we had to accept the pathologists' reports at each site.

Despite these limitations, our study has several strengths. We have analyzed a large cohort of pediatric and adult EoE patients derived from the food allergy referral centers associated with CoFAR. The sample size, patient heterogeneity and extensive systematically collected information provided a unique opportunity for disease assessment, which will also be correlated in the future with gene sequencing data to understand how the described disease characteristics vary as a function of genetics. Notable findings from our current analysis 
included a persistent gap between symptom onset and diagnosis, influenced not only by age and race but also by presence of food allergy and atopic dermatitis. The interplay of these factors needs to be further investigated. Diagnostic delay after symptom onset was longer in white individuals and adults, findings that illustrate unique presentations dependent upon race and age and thus inform clinical practice and directions for future research. In addition, we found symptom variability with age and race, suggesting the need to elucidate the reasons for these differences. Of equal importance are the high prevalence of failure to thrive and depression/anxiety, which emphasize the need for a more comprehensive multidisciplinary care for patients with EoE. Other notable findings included the coexistence of extraesophageal eosinophilic gastrointestinal inflammation, multiple atopic diatheses and concurrent autoimmune diseases in EoE, as well as enrichment of EoE/EGID in first-degree relatives. Although it is possible that the high prevalence of co-morbid atopic disorders was in part influenced by heavy recruitment from allergy clinics, EoE is a disease that requires co-management by an allergist regardless of allergic comorbidities, and therefore less likely to bias our findings. We also confirmed that esophageal rings and strictures at diagnosis were significantly more common in adults, and underscore the need for early recognition and treatment. Esophageal eosinophilia was still significant in these patients, arguing for the use of medical therapies rather than contending with esophageal dilatations. Finally, analysis of co-morbidities in our cohort reveals the need for educational efforts to be aimed at a wider range of health care providers to consider a work-up for EoE, and to include those patients not only with esophageal symptoms, but also with various gastrointestinal symptoms.

\section{Supplementary Material}

Refer to Web version on PubMed Central for supplementary material.

\section{Acknowledgments}

This study would not be possible without the additional CoFAR site investigators: Tamara T. Perry, MD; Amy Scurlock, MD; Edwin Kim, MD; Evan Dellon, MD, MPH; and Donald Stablein, PhD. Other people who contributed to this study include Jessica Gau, RN, PNP; Diane Ananos, RN; Zara Atal, BS; Kim Mudd, RN, BSN, MS; Thomas Fitzgerald, BS; Peggy Chandler, APN; Erin O’Brien, BS; Sarah Beckwith, BS; Suzanne Carlisle, RN; Anne Hiegel, RN; Darlene Bailey, RN, Pam Steele, BSN; Jessica Gebhart, MSHS; Deanna Hamilton, RN; Wendy Moore MS; JoAnne Newton RN; Susan Leung, RN; Jill Slinkes, RN; Donna Brown; Marshall Plaut, MD; and Julian Poyser, MPA, MS, CRNP.

Funding: Sources of support: NIH-NIAID U19AI066738 and U01AI066560. The project was also supported by Grant Numbers UL1 RR-025780 (National Jewish), UL1 TR000067 (Mount Sinai), UL1 TR-000039 (Arkansas), UL1 TR-000083 (U North Carolina) and UL1 TR-000424 (Johns Hopkins) from the National Center for Research Resources (NCRR), a component of the National Institutes of Health (NIH). Its contents are solely the responsibility of the authors and do not necessarily represent the official view of NCRR or NIH. Other funding sources: LaCache Fund for GI Allergic and Immunologic Diseases.

\section{Abbreviations}

CoFAR

EGID
Consortium for Food Allergy Research

eosinophilic gastrointestinal disorder

Eosinophilic esophagitis 


$\begin{array}{ll}\text { HPF } & \text { high power field } \\ \text { IQR } & \text { Interquartile range } \\ \text { GER } & \text { gastroesophageal reflux } \\ \text { GERD } & \text { gastroesophageal reflux disease } \\ \text { GI } & \text { gastrointestinal } \\ \text { PPI } & \text { proton pump inhibitor } \\ \text { PPI-REE } & \text { PPI-responsive esophageal eosinophilia } \\ \text { US } & \text { United States }\end{array}$

\section{References}

1. Liacouras CA, Furuta GT, Hirano I, Atkins D, Attwood SE, Bonis PA, et al. Eosinophilic esophagitis: updated consensus recommendations for children and adults. J Allergy Clin Immunol. 2011; 128(1):3-20. e6. quiz 1-2. [PubMed: 21477849]

2. Kagalwalla AF, Wechsler JB, Amsden K, Schwartz S, Makhija M, Olive A, et al. Efficacy of a 4Food Elimination Diet for Children With Eosinophilic Esophagitis. Clin Gastroenterol Hepatol. 2017; 15(11):1698-707. e7. [PubMed: 28603055]

3. Almansa C, Krishna M, Buchner AM, Ghabril MS, Talley N, DeVault KR, et al. Seasonal distribution in newly diagnosed cases of eosinophilic esophagitis in adults. Am J Gastroenterol. 2009; 104(4):828-33. [PubMed: 19240704]

4. Dellon ES. Epidemiology of eosinophilic esophagitis. Gastroenterology clinics of North America. 2014; 43(2):201-18. [PubMed: 24813510]

5. Spergel JM, Brown-Whitehorn TF, Beausoleil JL, Franciosi J, Shuker M, Verma R, et al. 14 years of eosinophilic esophagitis: clinical features and prognosis. J Pediatr Gastroenterol Nutr. 2009; 48(1): 30-6. [PubMed: 19172120]

6. Assa'ad AH, Putnam PE, Collins MH, Akers RM, Jameson SC, Kirby CL, et al. Pediatric patients with eosinophilic esophagitis: an 8-year follow-up. J Allergy Clin Immunol. 2007; 119(3):731-8. [PubMed: 17258309]

7. Kottyan LC, Davis BP, Sherrill JD, Liu K, Rochman M, Kaufman K, et al. Genome-wide association analysis of eosinophilic esophagitis provides insight into the tissue specificity of this allergic disease. Nat Genet. 2014; 46(8):895-900. [PubMed: 25017104]

8. Furuta GT, Liacouras CA, Collins MH, Gupta SK, Justinich C, Putnam PE, et al. Eosinophilic esophagitis in children and adults: a systematic review and consensus recommendations for diagnosis and treatment. Gastroenterology. 2007; 133(4):1342-63. [PubMed: 17919504]

9. Lucendo AJ, Arias A, Gonzalez-Cervera J, Olalla JM, Molina-Infante J. Dual response to dietary/ topical steroid and proton pump inhibitor therapy in adult patients with eosinophilic esophagitis. J Allergy Clin Immunol. 2016; 137(3):931-4. e2. [PubMed: 26371836]

10. Wen T, Dellon ES, Moawad FJ, Furuta GT, Aceves SS, Rothenberg ME. Transcriptome analysis of proton pump inhibitor-responsive esophageal eosinophilia reveals proton pump inhibitor-reversible allergic inflammation. J Allergy Clin Immunol. 2015; 135(1):187-97. [PubMed: 25441638]

11. Shoda T, Matsuda A, Nomura I, Okada N, Orihara K, Mikami H, et al. Eosinophilic esophagitis versus proton pump inhibitor-responsive esophageal eosinophilia: Transcriptome analysis. J Allergy Clin Immunol. 2017; 139(6):2010-3. e4. [PubMed: 28063872]

12. Bai JC, Fried M, Corazza GR, Schuppan D, Farthing M, Catassi C, et al. World Gastroenterology Organisation global guidelines on celiac disease. J Clin Gastroenterol. 2013; 47(2):121-6. [PubMed: 23314668] 
13. Jacobson DL, Gange SJ, Rose NR, Graham NM. Epidemiology and estimated population burden of selected autoimmune diseases in the United States. Clin Immunol Immunopathol. 1997; 84(3): 223-43. [PubMed: 9281381]

14. Weinberger AH, Gbedemah M, Martinez AM, Nash D, Galea S, Goodwin RD. Trends in depression prevalence in the USA from 2005 to 2015: widening disparities in vulnerable groups. Psychol Med. 2017:1-10.

15. Restani P, Ballabio C, Tripodi S, Fiocchi A. Meat allergy. Curr Opin Allergy Clin Immunol. 2009; 9(3):265-9. [PubMed: 19369863]

16. Park JR, Pfeil SA. Primary Care of the Patient with Inflammatory Bowel Disease. Med Clin North Am. 2015; 99(5):969-87. [PubMed: 26320042]

17. Moawad FJ, Dellon ES, Achem SR, Ljuldjuraj T, Green DJ, Maydonovitch CL, et al. Effects of Race and Sex on Features of Eosinophilic Esophagitis. Clin Gastroenterol Hepatol. 2016; 14(1): 23-30. [PubMed: 26343181]

18. Jensen ET, Martin CF, Kappelman MD, Dellon ES. Prevalence of Eosinophilic Gastritis, Gastroenteritis, and Colitis: Estimates From a National Administrative Database. J Pediatr Gastroenterol Nutr. 2016; 62(1):36-42. [PubMed: 25988554]

19. Ko HM, Morotti RA, Yershov O, Chehade M. Eosinophilic gastritis in children: clinicopathological correlation, disease course, and response to therapy. Am J Gastroenterol. 2014; 109(8):1277-85. [PubMed: 24957155]

20. Caldwell JM, Collins MH, Stucke EM, Putnam PE, Franciosi JP, Kushner JP, et al. Histologic eosinophilic gastritis is a systemic disorder associated with blood and extragastric eosinophilia, TH2 immunity, and a unique gastric transcriptome. J Allergy Clin Immunol. 2014; 134(5):111424. [PubMed: 25234644]

21. Kaur S, Rosen JM, Kriegermeier AA, Wechsler JB, Kagalwalla AF, Brown JB. Utility of Gastric and Duodenal Biopsies During Follow-up Endoscopy in Children With Eosinophilic Esophagitis. J Pediatr Gastroenterol Nutr. 2017; 65(4):399-403. [PubMed: 28118289]

22. Schoepfer AM, Straumann A, Panczak R, Coslovsky M, Kuehni CE, Maurer E, et al. Development and validation of a symptom-based activity index for adults with eosinophilic esophagitis. Gastroenterology. 2014; 147(6):1255-66. e21. [PubMed: 25160980]

23. Giriens B, Yan P, Safroneeva E, Zwahlen M, Reinhard A, Nydegger A, et al. Escalating incidence of eosinophilic esophagitis in Canton of Vaud, Switzerland, 1993-2013: a population-based study. Allergy. 2015; 70(12):1633-9. [PubMed: 26304142]

24. Chehade M, Sampson HA, Morotti RA, Magid MS. Esophageal subepithelial fibrosis in children with eosinophilic esophagitis. J Pediatr Gastroenterol Nutr. 2007; 45(3):319-28. [PubMed: 17873744]

25. Katzka DA, Geno DM, Blair HE, Lamsam JL, Alexander JA, Camilleri M. Small intestinal permeability in patients with eosinophilic oesophagitis during active phase and remission. Gut. 2015; 64(4):538-43. [PubMed: 24957264]

26. Harris RF, Menard-Katcher C, Atkins D, Furuta GT, Klinnert MD. Psychosocial dysfunction in children and adolescents with eosinophilic esophagitis. J Pediatr Gastroenterol Nutr. 2013; 57(4): 500-5. [PubMed: 23752077]

27. Franciosi JP, Hommel KA, DeBrosse CW, Greenberg AB, Greenler AJ, Abonia JP, et al. Quality of life in paediatric eosinophilic oesophagitis: what is important to patients? Child Care Health Dev. 2012; 38(4):477-83. [PubMed: 21671982]

28. van Rhijn BD, Smout AJ, Bredenoord AJ. Disease duration determines health related quality of life in adult eosinophilic esophagitis patients. Neurogastroenterol Motil. 2014; 26(6):772-8. [PubMed: 24602119]

29. Heifert TA, Susi A, Hisle-Gorman E, Erdie-Lalena CR, Gorman G, Min SB, et al. Feeding Disorders in Children With Autism Spectrum Disorders Are Associated With Eosinophilic Esophagitis. J Pediatr Gastroenterol Nutr. 2016; 63(4):e69-73. [PubMed: 27276430]

30. Namjou B, Marsolo K, Caroll RJ, Denny JC, Ritchie MD, Verma SS, et al. Phenome-wide association study (PheWAS) in EMR-linked pediatric cohorts, genetically links PLCL1 to speech language development and IL5-IL13 to Eosinophilic Esophagitis. Front Genet. 2014; 5:401. [PubMed: 25477900] 
31. Lucendo AJ, Arias A, Tenias JM. Systematic review: the association between eosinophilic oesophagitis and coeliac disease. Aliment Pharmacol Ther. 2014; 40(5):422-34. [PubMed: 25041372]

32. Peterson K, Firszt R, Fang J, Wong J, Smith KR, Brady KA. Risk of Autoimmunity in EoE and Families: A Population-Based Cohort Study. Am J Gastroenterol. 2016; 111(7):926-32. [PubMed: 27215923]

33. Arora M, Bagi P, Strongin A, Heimall J, Zhao X, Lawrence MG, et al. Gastrointestinal Manifestations of STAT3-Deficient Hyper-IgE Syndrome. J Clin Immunol. 2017; 37(7):695-700. [PubMed: 28803389]

34. Chen M, Ko HM, Riffle ME, Andreae DA, Cunningham-Rundles C, Chehade M, et al. Eosinophilic esophagitis diagnosed in a patient with common variable immunodeficiency. $\mathrm{J}$ Allergy Clin Immunol Pract. 2016; 4(5):995-7. [PubMed: 27157654]

35. Lucendo AJ, Molina-Infante J, Arias A, von Arnim U, Bredenoord AJ, Bussmann C, et al. Guidelines on eosinophilic esophagitis: evidence-based statements and recommendations for diagnosis and management in children and adults. United European Gastroenterol J. 2017; 5(3): 335-58.

36. Molina-Infante J, Bredenoord AJ, Cheng E, Dellon ES, Furuta GT, Gupta SK, et al. Proton pump inhibitor-responsive oesophageal eosinophilia: an entity challenging current diagnostic criteria for eosinophilic oesophagitis. Gut. 2016; 65(3):524-31. [PubMed: 26685124]

37. Cheng E, Zhang X, Huo X, Yu C, Zhang Q, Wang DH, et al. Omeprazole blocks eotaxin-3 expression by oesophageal squamous cells from patients with eosinophilic oesophagitis and GORD. Gut. 2013; 62(6):824-32. [PubMed: 22580413]

38. Sodikoff J, Hirano I. Proton pump inhibitor-responsive esophageal eosinophilia does not preclude food-responsive eosinophilic esophagitis. J Allergy Clin Immunol. 2016; 137(2):631-3. [PubMed: 26318073] 


\section{Highlights box}

\section{What is known about this topic?}

EoE is increasingly common, but data on its phenotypic aspects are still incomplete.

\section{What does this article add to our knowledge?}

An age and race-dependent variation in endoscopic features, symptoms and time gap to diagnosis is present. Concurrent atopic diseases, failure to thrive and depression/anxiety are prevalent in patients with EoE. Esophageal eosinophilia is present even when the esophagus has fibrostenotic features.

\section{How does this study impact current management guidelines?}

Physician education is important regarding symptom variability. A multi-disciplinary approach is needed for better care of EoE patients given the co-morbidities. 


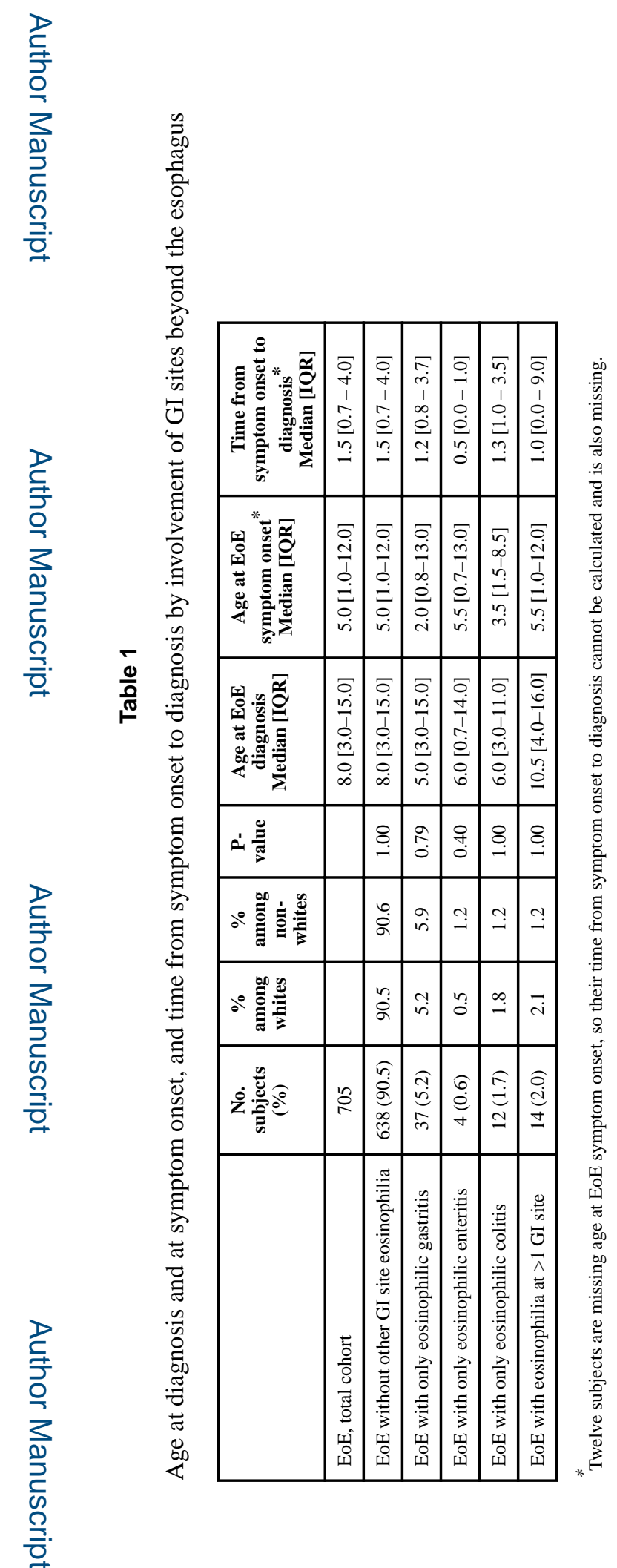

J Allergy Clin Immunol Pract. Author manuscript; available in PMC 2019 September 01. 


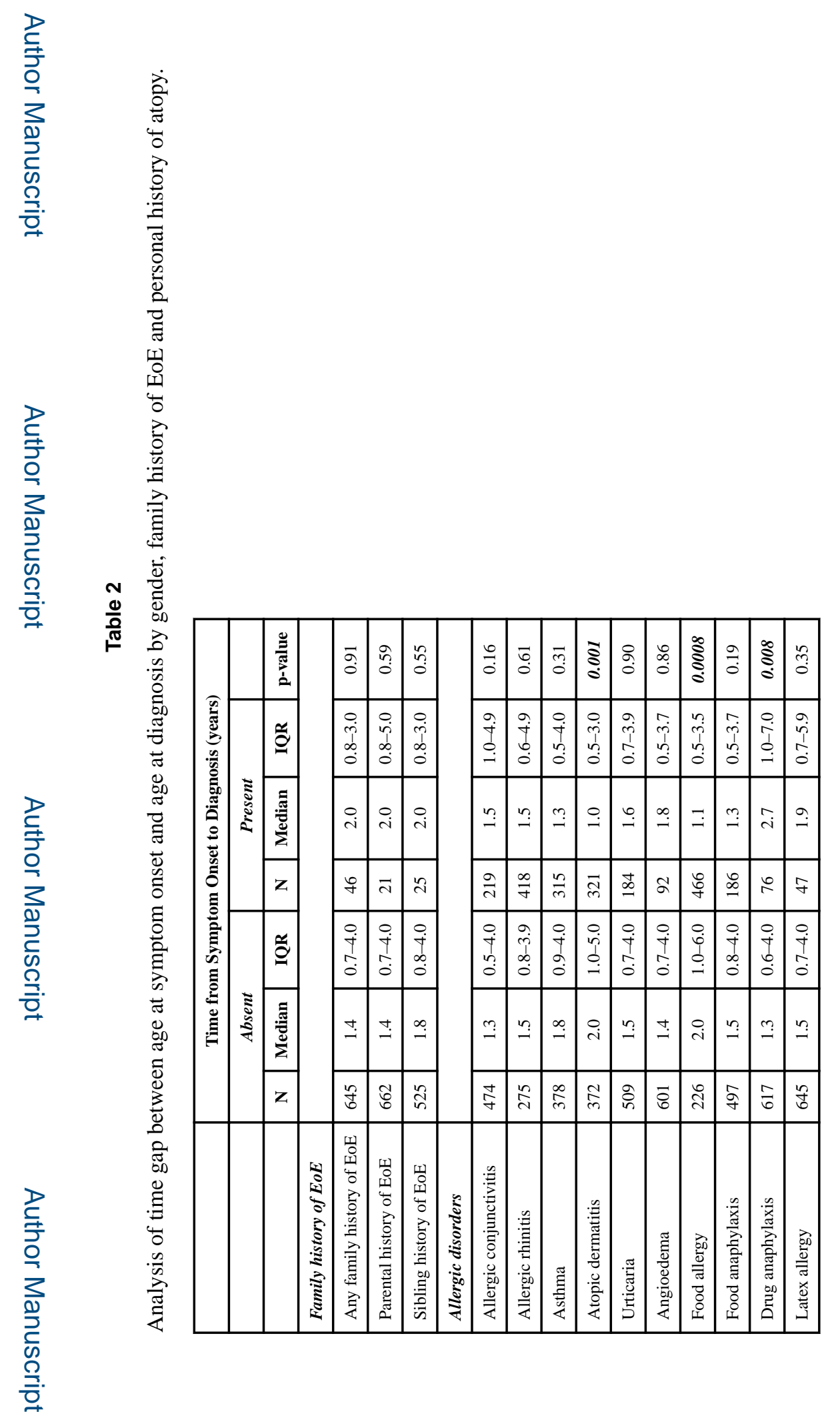

J Allergy Clin Immunol Pract. Author manuscript; available in PMC 2019 September 01. 


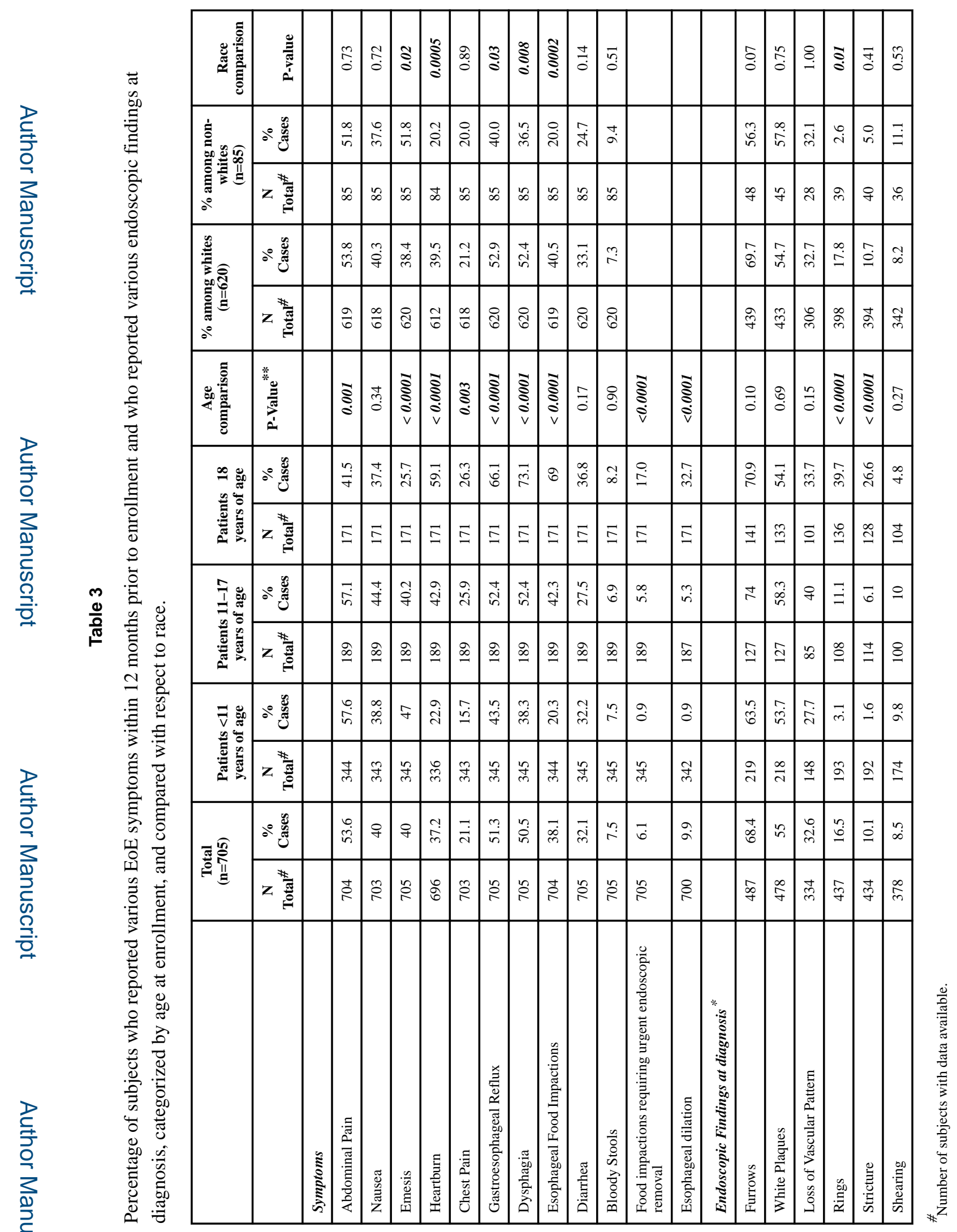

J Allergy Clin Immunol Pract. Author manuscript; available in PMC 2019 September 01. 


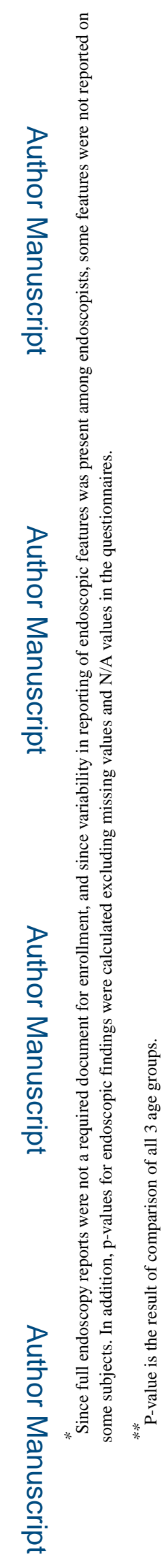




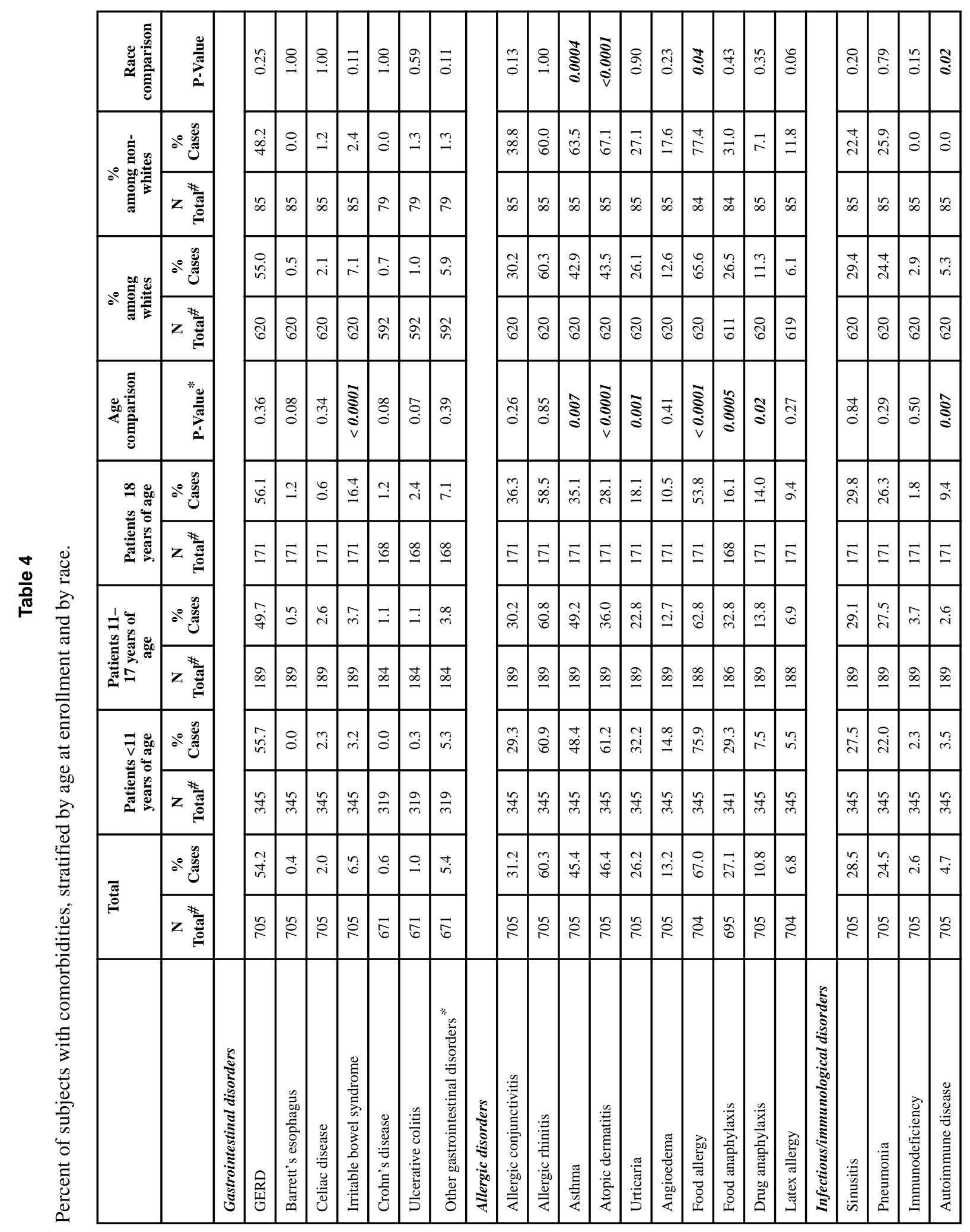

J Allergy Clin Immunol Pract. Author manuscript; available in PMC 2019 September 01. 


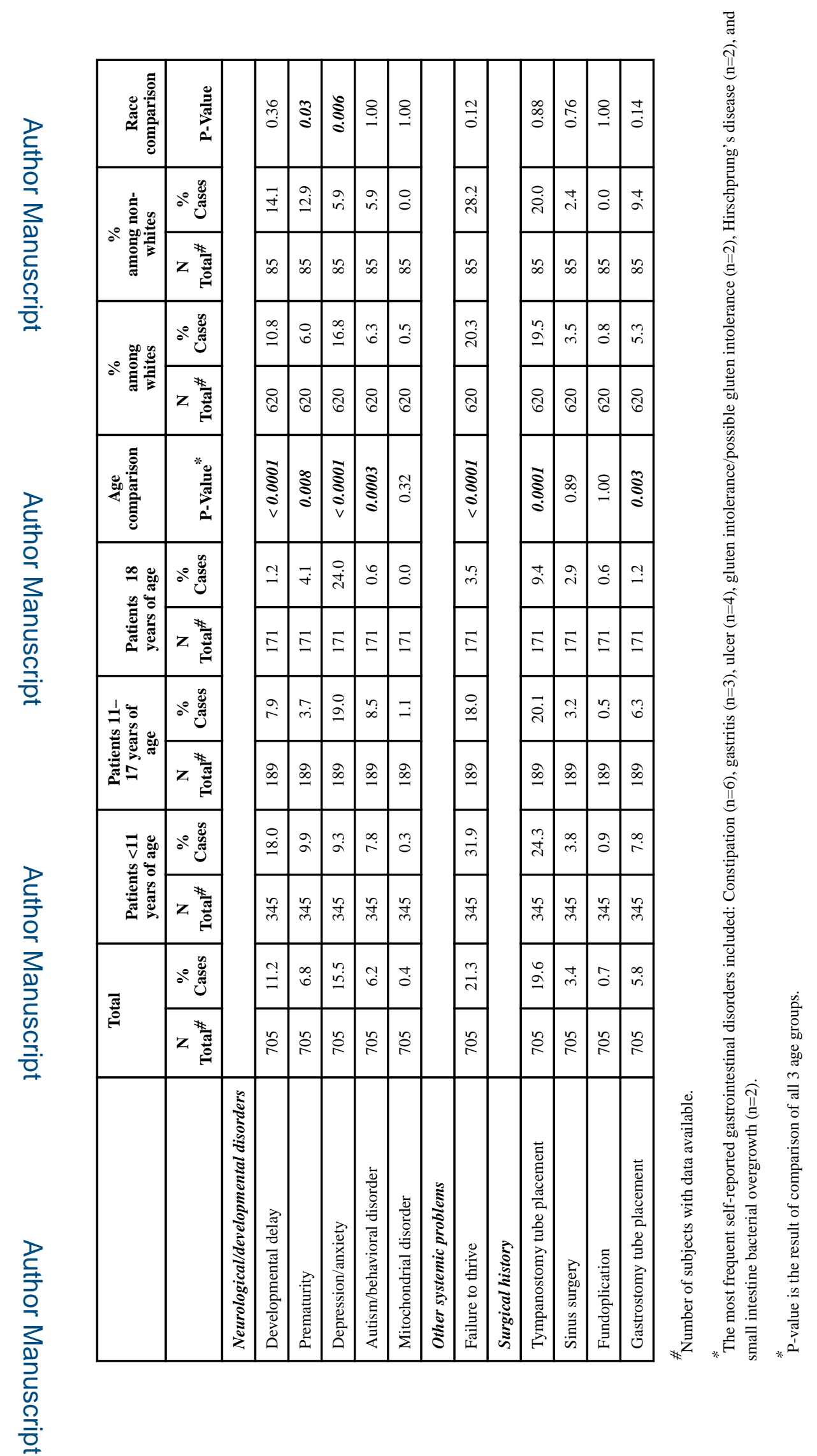

J Allergy Clin Immunol Pract. Author manuscript; available in PMC 2019 September 01. 